\title{
Quality improvement initiatives in fragility fracture care and prevention
}

Keywords: Fracture Liaison Service; Orthogeriatric Services; secondary fracture prevention; systemsbased approach; fracture prevention policy; Capture the Fracture ${ }^{\circledR}$; osteoporosis; fragility fracture; International Osteoporosis Foundation; Fragility Fracture Network, American Society for Bone and Mineral Research; Asia Pacific Fragility Fracture Alliance

Corresponding Author: Paul J. Mitchell

Contact Details:

Tel: $+64(0) 98893127$

Cell: $+64(0) 212710128$

Email: paul.mitchell@ndorms.ox.ac.uk

Address:

c/o 3 Harris Street

Pukekohe

2120

New Zealand

Paul J. Mitchell ${ }^{1,2,3}$, Cyrus Cooper ${ }^{4,5,6,7}$, Masaki Fujita ${ }^{7}$, Philippe Halbout ${ }^{7}$, Kristina Åkesson ${ }^{7,8,9}$, Matthew Costa $^{3,10}$, Karsten E. Dreinhöfer ${ }^{3,11,12}$, David R. Marsh ${ }^{3,13}$, Joon-Kiong Lee ${ }^{14,15}$, Ding-Cheng (Derrick) Chan $^{16,17}$, M. Kassim Javaid ${ }^{7,18}$

Affiliations:

1. Nuffield Department of Orthopaedics, Rheumatology and Musculoskeletal Sciences, University of Oxford, Oxford, UK.

2. School of Medicine, Sydney Campus, The University of Notre Dame Australia, 140 Broadway, Sydney, NSW, 2007, Australia.

3. Fragility Fracture Network, c/o MCI Schweiz AG, Schaffhauserstrasse 550, 8052, Zürich, Switzerland.

4. MRC Lifecourse Epidemiology Unit, University of Southampton, Southampton, UK.

5. NIHR Southampton Biomedical Research Centre, University of Southampton and University Hospital Southampton NHS Foundation Trust, Southampton, UK.

6. NIHR Oxford Biomedical Research Centre, University of Oxford, Oxford, UK.

7. International Osteoporosis Foundation, 9 rue Juste-Olivier, $\mathrm{CH}-1260$, Nyon, Switzerland.

8. Department of Orthopaedics, Skane University Hospital, Malmö, Sweden.

9. Lund University, Department of Clinical Sciences, Malmö, Sweden.

10. Oxford Trauma, Nuffield Department of Orthopaedics, Rheumatology and Musculoskeletal Sciences, University of Oxford, Oxford, UK. 
11. Department of Musculoskeletal Rehabilitation, Prevention and Health Service Research, Center for Sport Science and Sport Medicine (CSSB), Center for Musculoskeletal Surgery (CMSC), Charité Universitätsmedizin, Berlin, Germany.

12. Department of Orthopedics and Traumatology, Medical Park Berlin Humboldtmühle, Berlin, Germany.

13. University College London, Gower St, Bloomsbury, London, WC1E 6BT, UK.

14. Department of Orthopedic Surgery, Beacon International Specialist Centre, Petaling Jaya, Selangor, Malaysia.

15. Advanced Neuroscience and Orthopedic Centre (ANOC), Kuala Lumpur, Malaysia.

16. Department of Geriatrics and Gerontology and Department of Internal Medicine, National Taiwan University Hospital, Taipei, Taiwan.

17. Superintendent Office, Chutung Branch, National Taiwan University Hospital, Hsinchu County, Taiwan.

18. Oxford NIHR Biomedical Research Centre, University of Oxford, Oxford, UK. 


\begin{abstract}
Purpose of review: This review sought to describe quality improvement initiatives in fragility fracture care and prevention.

Recent findings: A major care gap persists throughout the world in the secondary prevention of fragility fractures. Systematic reviews have confirmed that the Fracture Liaison Service (FLS) model of care is associated with significant improvements in rates of bone mineral density testing, initiation of osteoporosis treatment and adherence with treatment for individuals who sustain fragility fractures. Further, these improvements in the processes of care resulted in significant reductions in refracture risk and lower post-fracture mortality. The primary challenge facing health systems now is to ensure that best practice is delivered effectively in the local healthcare setting. Publication of clinical standards for FLS at the organisational and patient level in combination with the establishment of national registries has provided a mechanism for FLS to benchmark and improvement their performance.
\end{abstract}

Summary: Major efforts are ongoing at the global, regional and national level to improve the acute care, rehabilitation and secondary prevention for individuals who sustain fragility fractures. Active participation in these initiatives has the potential to eliminate current care gaps in the coming decade. 


\section{Introduction}

Humankind is entering a new demographic era. In 2017, the United Nations (UN) Department of Economic and Social Affairs reported the population of the world to be 7.6 billion people [1]. The proportion of children aged $<15$ years $(26 \%)$ is currently twice that of the proportion of adults aged $\geq 60$ years (13\%). However, this ratio will change dramatically during the remainder of this century. The pace and extent of this change can be illustrated by the age dependency ratios described in the UN population projections. The so-called "old-age" dependency ratio is the ratio of the population aged $\geq 65$ years to the population aged 15-64 years, who are considered to be of "working age". These ratios are presented as the number of dependents per 100 persons of working age [2]. In figure 1, the old-age dependency ratios for the world and major world regions are shown for the period 1950 to 2100. Except for Africa, the populations of all world regions are currently undergoing a major shift in their respective age structures. As a consequence of this, the G20 group of nations has recently identified ageing as a global risk [3]. Among wealthy nations, the combination of increasing life-expectancy and falling birth rates has resulted in a rapid expansion of the elderly population. South Korea is a notable example, where the "longevity miracle" is projected to result in the old-age dependency ratio increasing from 10 retirees per 100 people of working age at the turn of the century to 66.3 by 2050 [2]. A direct consequence of this mass ageing of the global population will be a dramatic increase in the prevalence of chronic diseases which affect older people, including osteoporosis and the fragility fractures that result from the condition.

Please insert Figure 1 here

In 2017, the International Osteoporosis Foundation (IOF) published the first edition of the IOF Compendium of Osteoporosis which provides a comprehensive overview of the burden of osteoporosis globally and in the world regions [4]. The Compendium highlighted that the current incidence of fragility fractures is very high and is set to increase dramatically as the world's population ages:

- Asia-Pacific: By 2050, 1.3 billion people in Asia will be aged 60 years or older and more than a quarter of a billion will be aged 80 years or older [5]. The annual incidence of hip fracture in China is set to rise from 411,000 cases in 2015 to 1 million cases in 2050 [6].

- Europe: In 2010, the 3.5 million fragility fractures which occurred in the European Union contributed to the total cost of osteoporosis reaching Euro 37 billion (US\$40 billion) [7].

- Latin America: The most rapidly ageing region of the world between 2015 and 2030 [8]. In Brazil, the number of hip fractures is projected to more than double, from 80,640 cases in 2015 to 198,000 cases by 2040 [9].

- North America: By 2025, the annual incidence of fragility fractures in the United States is projected to exceed 3 million cases, at a cost of US\$25 billion [10]. 


\section{Secondary fracture prevention: a worldwide, persistent and pervasive care gap}

Individuals who have sustained fragility fractures provide an identifiable group that are at high imminent risk of sustaining second and subsequent fractures. Meta-analyses have demonstrated that a prior fracture at any site is associated with a doubling of future fracture risk [11, 12]. From the obverse perspective, we have known since the 1980s that up to half of hip fracture patients have a history of prior fractures in the months or years before they broke their hip [13-16]. Furthermore, numerous clinical practice guidelines for the management of osteoporosis identify fragility fractures as a primary risk factor for osteoporosis. However, almost a quarter of a century after the introduction of pharmacological treatments for osteoporosis, which have been shown to significantly reduce the incidence of secondary fractures [17], most fragility fracture patients worldwide do not receive guidelines-based care. In 2016, IOF described the care gap in the World Osteoporosis Day thematic report based on a broad range of studies from countries in Asia, Europe, the Middle East, North America and Oceania [18].

\section{Closing the secondary fracture prevention care gap: The role of Fracture Liaison Services}

During the late 1990s and early 2000s, investigators from Australia [19], Canada [20], United Kingdom [21] and United States of America [22] developed what would become known as the Fracture Liaison Service (FLS) model of care to address the secondary prevention care gap. The purpose of a FLS is to ensure that all patients aged 50 years or over, who present to urgent care services with a fragility fracture, undergo fracture risk assessment and receive treatment in accordance with prevailing national clinical guidelines for osteoporosis. The FLS also ensures that falls risk is addressed among older patients through referral to appropriate local falls prevention services.

In 2013, Ganda and colleagues undertook a systematic review of publications which described models of care intended to improve rates of secondary fracture prevention [23]. On account of the heterogeneity of the fashion in which the various models were organised, a classification system was developed which designated FLS as Type A through to Type D:

- Type A FLS models: Identifies fracture patients, organises investigations and initiates osteoporosis treatment, where appropriate, for fragility fracture patients. A " $3 i$ " FLS model.

- Type B FLS models: Identifies and investigates but leaves the initiation of treatment to the primary care provider (PCP). A " $2 \mathrm{i}$ " FLS model.

- Type C FLS models: Fracture patients receive education about osteoporosis and receive lifestyle advice including falls prevention. The patient is recommended to seek further assessment and the PCP is alerted that the patient has sustained a fracture and that further assessment is needed. This model does not undertake BMD testing or assessment of need for osteoporosis treatment. A "1i" FLS model.

- Type D FLS models: Provides osteoporosis education to the fracture patient. Type D models do not educate or alert the primary care provider. A "Zero i" FLS model.

A meta-analysis of the primary studies was undertaken to determine the proportion of fracture patients who underwent bone mineral density (BMD) testing and received osteoporosis treatment for each of the four types of FLS model. Almost four-fifths (79\%) of patients managed by Type A FLS models 
underwent BMD testing and almost a half (46\%) received osteoporosis treatment. Type D FLS models resulted in less than a tenth (8\%) of fracture patients receiving osteoporosis treatment, while the Type $B$ and Type C FLS models achieved levels of BMD testing and treatment that were in between the Type A and Type D models.

In 2019, Ganda and colleagues published an update of the systematic review and meta-analysis to include studies published on secondary fracture prevention during the period 1996 to 2017 as a chapter [24] in a book which provides an international perspective on secondary fracture prevention [25]. In terms of initiation of osteoporosis treatments, the updated meta-analysis reported the following:

- Type A versus Type C FLS models: A risk difference of 0.29 (95\% Cl 0.26-0.32, p<0.001) was calculated indicating a $29 \%$ absolute difference in treatment initiation rates between the two models (favouring the Type A model). This was consistent with the difference between treatment rates in the Type $A$ intervention group versus the control group that was reported in the original meta-analysis (28.5\%) [23].

- Type B FLS models versus usual care: A risk difference of $0.16(95 \% \mathrm{Cl} 0.12-0.21, \mathrm{p}<0.001)$ was calculated indicating a $16 \%$ absolute difference in treatment initiation rates between the Type $B$ FLS model and usual care (favouring the Type B model). This was similar to the difference between treatment rates in the Type $B$ intervention group versus the control group that was reported in the original meta-analysis (20.7\%) [23].

- Type C FLS models versus usual care: A risk difference of 0.13 (95\% Cl 0.09-0.16, p<0.001) was calculated indicating a $13 \%$ absolute difference in treatment initiation rates between the Type $C$ FLS model and usual care (favouring the Type $\mathrm{C}$ model). This was similar to the difference between treatment rates in the Type $C$ intervention group versus the control group that was reported in the original meta-analysis (15.9\%) [23].

A key point is that the original 2013 publication by Ganda and colleagues did not include a meta-analysis of adherence and refracture rates due to an inadequate number of studies reporting these outcomes [23]. While there is commentary on FLS-related studies and adherence rates in the updated publication [24], meta-analysis of adherence rates was also not presented. This is a limitation of the studies, given the known low adherence to therapy [26] and specific inclusion in a number of publications including the IOF Capture the Fracture ${ }^{\circledR}$ Best Practice Framework [27] and UK national FLS audit key performance indicator [28]. The focus on treatment initiation without monitoring metrics is insufficient as it is poor predictor of treatment adherence at 12 and 24 months [29, 30].

In 2018, Wu and colleagues undertook a systematic review and meta-analysis of the FLS literature [26]. The meta-analysis revealed significant improvements in all reported outcomes for patients managed by a FLS compared to non-FLS controls:

- $\quad$ BMD testing was increased by $24 \%$ (95\% $\mathrm{Cl} 0.18-0.29)$.

- Osteoporosis treatment was increased by $20 \%(95 \% \mathrm{Cl} 0.16-0.25)$.

- Treatment adherence was increased by $22 \%(95 \% \mathrm{Cl} 0.13-0.31)$.

- Absolute risk of refracture was reduced by $5 \%(95 \% \mathrm{Cl}-0.08--0.03)$.

- Mortality was reduced by $3 \%(95 \% \mathrm{Cl}-0.05--0.01)$.

The authors state that the absolute risk reductions for refracture and mortality of $5 \%$ and $3 \%$, respectively, represent about $30 \%$ and $20 \%$ relative risk reductions in the incidence of refracture or 
death, respectively. In terms of relative risk reduction of refracture, this is similar to the $28 \%$ reduction of any clinical fracture reported in the vertebral fracture arm of the Fracture Intervention Trial which evaluated the efficacy of alendronate [31].

Recently, investigators from the Netherlands described opportunities and barriers to increase attendance at FLS by individuals who sustain fragility fractures [32]. Non-attendance was associated with the following factors:

- Male gender (Odds ratio [OR]: $2.08,95 \% \mathrm{Cl}: 1.35,3.21$ )

- Frailty (OR: $1.62, \mathrm{Cl}: 1.08,2.45)$

- $\quad$ Living alone (OR:2.05, $\mathrm{Cl}: 1.48,2.85)$

- Low education (OR: $1.82, \mathrm{Cl}: 1.27,2.63)$

- Not interested in bone strength (OR: $1.85, \mathrm{Cl}: 1.33,2.63$ )

- Being unaware of increased subsequent fracture risk (OR: $1.75, \mathrm{Cl}: 1.08,2.86$ )

However, adequately perceived advice to undergo bone densitometry testing and attend the FLS was strongly associated with FLS attendance (OR: $3.32, \mathrm{Cl}: 1.75,6.27)$. The authors propose that attendance could be increased by paying close attention the fracture registration process and by tailoring interactions with each patient to motivate their engagement.

The international perspective book includes detailed summaries of progress and barriers to implementation of systematic approaches to secondary fracture prevention in Australia, Canada, Japan, Lebanon, New Zealand, Singapore, Taiwan, the United Kingdom and the United States [25]. The strategies employed to drive clinical engagement and policy change may prove useful to colleagues in other countries and regions. Summaries follow of the significant progress that has been made in New Zealand, Taiwan and the UK in recent years. Furthermore, recent progress in several Scandinavian countries is also summarised.

\section{Progress in New Zealand}

In 2012, Osteoporosis New Zealand published a strategy document which proposed development of a systematic approach to hip fracture care and prevention for the country [33]. During the ensuring four years a multidisciplinary, multisector effort culminated in a major investment by the Accident Compensation Corporation (ACC), the Crown Entity responsible for managing a no-fault injury prevention scheme for all New Zealanders and visitors to New Zealand. ACC invested NZ\$30.5 million (US\$20.6 million) to support the nationwide implementation of the following initiatives for New Zealand's population of 5 million people:

- A national hip fracture registry to benchmark care of hip fracture patients against clinical standards.

- A FLS in every District Health Board.

- In-home and community-based strength and balance programmes.

- Assessment and management of visual acuity and environmental hazards in the home.

- Medication review for people taking multiple medicines.

- Vitamin D prescribing in Aged Residential Care. 
- Integrated services across primary and secondary care (including supported hospital discharge) to provide seamless pathways in the falls and fracture system.

In 2017, the multisector effort was formalised under the Live Stronger for Longer initiative, which is comprised of all relevant government agencies, NGOs and other stakeholders [34]. A Falls and Fractures Outcomes Framework has been developed to assess the impact of the activities described above [35]. The Outcomes Framework describes five domains which are populated with a range of measures pertaining to falls and fracture care, including quarterly data on the number of individuals seen by FLS and those participating in community-based strength and balances classes.

\section{Progress in Taiwan}

The Taiwanese National Health Insurance (NHI) system reimburses osteoporosis treatments for individuals who have sustained hip or vertebral fractures and have low BMD. Accordingly, the Taiwan FLS Network focuses on providing care for patients who have sustained these fractures [36]. The Taiwanese Osteoporosis Association (TOA) has been proactive in organising educational events to drive widespread adoption of FLS across Taiwan. Workshops and sharing of best practice between FLS sites have resulted in 24 FLS becoming operational in Taiwan by early 2019. The majority of these FLS ( $n=17)$ feature on the IOF Capture the Fracture ${ }^{\circledR}$ Map of Best Practice (which is discussed in detail below) [37]. In 2017, TOA-led initiative was recognised by IOF at the 2017 World Congress for Osteoporosis, Osteoarthritis and Musculoskeletal Diseases held in Florence and granted the Best Secondary Fracture Prevention Promotion award [38].

In January 2019, representatives of TOA engaged in negotiation with the administration of the National Health Insurance system with the aim of securing public reimbursement for FLS in Taiwan.

\section{Progress in the United Kingdom}

During the last two decades, national multi-sector, multidisciplinary efforts have been delivered in the UK to improve fragility fracture care and prevention as reported in a previous issue of this journal in 2013 [39]. Descriptions of more recent national initiatives follow.

\section{UK FLS Clinical Standards}

In 2015, the Royal Osteoporosis Society (ROS, previously the National Osteoporosis Society) published Clinical Standards for FLS [40]. The Clinical Standards were based upon the so-called " $5 I Q$ " approach, which defines standards relating to the following aspects of care: identification, investigation, information, intervention, integration and quality of service provision. The Clinical Standards were endorsed by the following organisations:

\footnotetext{
$>$ British Geriatric Society

British Menopause Society

$>$ British Orthopaedic Association

$>$ British Society for Rheumatology
} 
Chartered Society of Physiotherapy

$>$ International Osteoporosis Foundation

$>$ Royal College of General Practitioners

$>$ Royal College of Physicians

$>$ Royal Pharmaceutical Society

\section{Online FLS Resources}

ROS has also developed a comprehensive suite of online resources, including a FLS Benefits Calculator, and established a Service Development Team which is working with hospitals throughout the UK to improve access to, and performance of FLS [41].

\section{National FLS Database}

The Fracture Liaison Service Database (FLS-DB) was commissioned by the Healthcare Quality Improvement Partnership (HQIP) as a new national audit as part of the Fragility Fracture Audit Programme (FFFAP) delivered by the Royal College of Physicians (RCP) [42]. The FLS-DB is the first national FLS audit in the world and is comprised of two national audit components: a facilities audit and a patient audit. The first FLS-DB facilities audit [43] and patient audit [44] were published in 2016 and 2017, respectively. In December 2018, the FLS-DB 2018 Annual Report documented almost 53,000 patients records from 55 FLS from across England and Wales for the calendar year 2017 [28]. Key findings included:

- Identification: In 2017 , identification of all fractures had improved to $43 \%$ compared with $40 \%$ in 2016 . On average $6 \%$ of patients had a spine fracture in 2017 , compared with $4 \%$ in 2016.

- Intervention: The percentage of patients being recommended anti-osteoporosis medication increased to $43 \%$ in 2017 from $38 \%$ in 2016 . Forty six percent of patients received (or were referred for) a falls assessment compared with $40 \%$ in 2016.

- Monitoring: Monitoring declined during 2017, with only $38 \%$ of patients recommended antiosteoporosis medication being contacted at $12-16$ weeks post fracture compared with $41 \%$ in 2016.

Key recommendations were made with the intention of improving delivery of all key performance indicators.

By 2019, the FLS-DB held 200,000 patient records with 65 FLS actively participating. Ten of these FLS were successfully recruited into an Institute for Healthcare Improvement (IHI) Breakthrough Collaborative led by the RCP Quality Improvement Hub. This collaborative uses evidenced methods to upskill FLS practitioners in the science of quality improvement. This includes integration of patients into the service improvement model, identification of drivers for changes and data interpretation based on the FLS-DB live run charts [42] and provides a structure for FLS to participate using face-to-face meetings, coaching calls, online materials and pre and post user evaluations. 


\section{Progress in Scandinavia}

The Scandinavian countries are among those with the highest fracture incidence, hip fractures in particular. Although fracture epidemiology is described in detail, the implementation of preventive measures has been lagging until recently. In Sweden, national recommendations for secondary prevention was issued through the National Board of Health and Welfare in 2012, with a 2014 update emphasising the strong evidence for secondary prevention and FLS. Since then, locally adapted secondary prevention services have increased, most based in hospitals managing acute fractures.

Evaluation of services, also those requiring minimal resources have proved efficacious [45] and national network of FLS coordinators has been established. In Norway, a large scale multicentre FLS project, NoFRACT, based on the IOF Capture the Fracture ${ }^{\circledR}$ Program (described in detail below) is supported by national research grants to allow for evaluation [46]. The study aimed to include 26,000 patients, a number almost reached, with data on refracture rates [47]. In Denmark, FLS have been implemented at many sites and are also aiming to evaluate how to improve diagnostic precision and resource utilisation $[48,49]$.

\section{Global and regional implementation initiatives}

Several major global and regional initiatives to improve the acute care, rehabilitation and secondary fracture prevention of individuals who have sustained fragility fractures have been initiated in recent years. Descriptions of several of these initiatives follow in chronological order of their respective launch dates.

\section{IOF Capture the Fracture ${ }^{\circledR}$ Program}

Recognising the care gap for healthcare systems around the world to effectively deliver secondary fracture prevention, in 2012, IOF launched the Capture the Fracture ${ }^{\circledR}$ (CtF) Campaign with the aims of 1 ) being the global voice for secondary fracture prevention, 2) driving national and international policy, 3) ensuring quality and 4) providing support for FLS implementation, getting started, improving and sustainability. An international expert panel developed a Best Practice Framework (BPF) with 13 criteria and associated standards to assess the organisational effectiveness of FLS across the globe for the first time [50].

Central to the vision of IOF for a world without fragility fractures, the CtF Program provides the required infrastructure to support FLS implement the framework, including online resources and translations in over 8 languages [51]. The BPF demonstrated the feasibility of using a single tool to measure and compare the organisational performance of FLS in multiple healthcare settings [27]. Currently, there are over $340 \mathrm{FLS}$ from over 40 countries engaged with the programme and work is ongoing to renew existing sites which feature on the CtF Map of Best Practice (see figure 2) [37].

The next phase of support tools for FLS includes the IOF budget impact calculator and the "Getting to gold mentorship programme" [52]. The IOF budget impact calculator will determine the multiple benefits as well as the expected size and costs of running a FLS at local, regional and national levels, 
considering imminent fracture risk, therapeutic choices and other key characteristics in an open, transparent platform on the CtF website.

The major activity for the next three years for the CtF Programme will be the mentorship programme. The success of the CtF Program has created demand for ongoing support to develop effective FLS from stakeholders including industry (global and national), national healthcare systems, professional organisations and individual FLS that is only partially met by the existing CtF workshops and 1:1 peer mentoring activity. The "Getting to gold mentoring framework" is a bold and innovative step forward that formally aligns activities of the regional IOF offices, global and national industry partners, professional societies with the needs of established and nascent FLS champions in individual countries. The aim is to fast track implementation and development of effective, efficient FLS with a good patient experience by leveraging international experience and expertise. The programme will deliver across four pillars: a training scheme for a national cadre of trained FLS mentors, a national FLS policy and advocacy work package, a roadmap to support local FLS budget impact business cases and, crucially, local FLS support based on the theory and practice of quality improvement and mentoring.

The work completed so far by the CtF Program and planned activities going forward provides the key platform for quality improvement of FLS globally. This will be supported by the IOF Global Patient Charter which articulates the rights and responsibilities of all key stakeholders to ensure that the right patient receives the right treatment at the right time [53].

Please insert figure 2 here

\section{ASBMR Secondary Fracture Prevention Initiative}

In July 2017, the American Society for Bone and Mineral Research (ASBMR) supported a multistakeholder meeting in Virginia, USA with organisational support from the Center for Medical Technology Policy (CMTP), an independent non-profit organisation dedicated to evidence-based policy development. Pursuant to this meeting and informed by discussions among stakeholders, a draft strategic roadmap was subjected to consultation among all meeting participants and other learned organisations and advocacy groups throughout the world. In December 2017, the final version of the Strategic Roadmap to Prevent Secondary Fractures was published [54].

In 2018, ASBMR and CMTP formed a 40-member coalition of organisational representatives to develop a detailed action plan and consensus clinical recommendations. There was a consensus that the target population for the initiative would be those at very high risk of fracture: women and men, age 65 years or older, with a hip or vertebral fracture. Consensus clinical recommendations were developed with the overarching principle that they should be managed in the context of a multidisciplinary clinical system that includes case management (one example is a FLS) to assure that they are appropriately evaluated and treated for osteoporosis and risk of future fractures. A related media release issued during the 2018 ASBMR annual scientific meeting highlighted the need for individuals aged 65 years and over who sustain hip or vertebral fractures to receive treatment for osteoporosis [55]. A manuscript is currently in process for publication of the clinical recommendations.

Work on the Action Plan for the initiative is underway, with the following elements:

1. Promote clinical recommendations.

2. Establish national fracture registry. 
3. Increase number and capabilities of case management / FLS.

4. Improve diagnosis of and communication about patients with vertebral fractures.

5. Explore potential uses of reimbursement and financial incentives.

6. Continue to develop specific quantifiable goals and identify quality measures.

7. Stay abreast of evolution of clinical standards and explore potential uses of new technologies.

8. Continue to develop pilot programs for preventing secondary fracture for testing in integrated healthcare delivery systems.

9. Build relationships with key organizations that are not coalition members.

10. Continue coordination.

\section{Global Call to Action}

In September 2016, the global Fragility Fracture Network (FFN) convened a "Presidents' Roundtable" during the $5^{\text {th }}$ FFN Global Congress held in Rome. The purpose of the roundtable was to explore how several global and regional organisations could collaborate to improve all aspects of care of people presenting with fragility fractures. The organisations represented were:

- European Federation of National Associations of Orthopaedics and Traumatology (EFORT)

- European Geriatric Medicine Society (EuGMS)

- Fragility Fracture Network (FFN)

- International Collaboration of Orthopaedic Nursing (ICON)

- International Geriatric Fracture Society (IGFS)

- International Osteoporosis Foundation (IOF)

There was general agreement that despite enormous efforts to improve matters by the participating organisations and many other organisations throughout the world, insufficient improvement in the quality of care worldwide had been achieved. The participating organisations decided that the first step to address this challenge would be to prepare a multidisciplinary and multiprofessional Global Call to Action (CtA). In July 2018, the CtA was published and called for urgent improvements globally in the following three so-called clinical "pillars" [56]:

- Acute multidisciplinary care for the person who suffers a hip, clinical vertebral and other major fragility fractures.

- Rapid secondary prevention after first occurrence of all fragility fractures, including those in younger people as well as those in older persons, to prevent future fractures.

- Ongoing post-acute care of people whose ability to function is impaired by hip and major fragility fractures.

The CtA highlighted Orthogeriatric Services (aka orthopaedic-geriatric co-care programs), FLS and multidisciplinary rehabilitation teams as the optimal models of care to achieve these goals. Prior to publication, 81 organisations operating at the global level, regional level (Asia Pacific, Europe, Latin America, Middle East and Africa) and at the national level for five highly populated countries (Brazil, China, India, Japan and the United States of America) had endorsed the CtA.

The CtA called for specific actions from different sectors. For health professional societies these were: 
- To collaborate nationally and locally to form alliances to speak with a unified voice to policy makers.

- To produce consensus guidelines setting clear standards for adequate care using the best available research evidence and propose metrics to evaluate performance.

- To expand education and research programs that can establish best practice.

This "fourth pillar" of the CtA relates to the formation of national multidisciplinary alliances and is based on prior experience gained in several countries, notably Australia, Ireland, New Zealand and the UK, where the formation of such alliances has delivered significant patient benefit. The key metric of success for these national alliances will be their ability to translate the Call to Action into Actual Action.

\section{Asia Pacific Fragility Fracture Alliance}

In November 2018, the Asia Pacific Fragility Fracture Alliance (APFFA) was launched, which is comprised of the following regional and global member organisations [57]:

- Asian Federation of Osteoporosis Societies

- Asia-Oceanian Society of Physical and Rehabilitation Medicine

- Asia Pacific Geriatric Medicine Network

- Asia Pacific Orthopaedic Association

- Fragility Fracture Network

- International Osteoporosis Foundation

- International Society for Clinical Densitometry

The APFFA member organisations signed a multiparty Memorandum of Understanding (MoU) in late 2018. The primary purpose of APFFA is to drive policy change, improve awareness and change political and professional mindsets to facilitate optimal fragility fracture management across the Asia Pacific region. The clinical focus of APFFA is aligned to that of the Global Call to Action [56], namely, to improve acute care for people in the Asia Pacific region who sustain fragility fractures, subsequent patient rehabilitation and secondary fracture prevention.

\section{Conclusions}

Throughout the world, the demographic composition of our societies is changing at a very rapid rate. Consequently, maintaining the mobility and independence of our older people must be a key component of the global response to the ageing of humankind. Left unchecked, the burden imposed by osteoporosis and the fragility fractures that result from the condition will escalate and stretch our health systems - orthopaedics and care of older people departments, in particular - to, and potentially beyond the limit of their capacities. Implementation of a systematic approach to fragility fracture care and service improvement is urgently required if the impending surge in demand for acute fracture services is to be averted. Widespread implementation of the Fracture Liaison Service model of care will ensure that a determined effort is made to make the first fragility fracture the last. In parallel, efforts to deliver optimal acute care and rehabilitation for people who sustain serious fragility fractures will improve outcomes for patients and make for efficient use of precious hospital resources. 


\section{Research agenda}

1. FLS should seek to collect and publish more data on outcomes such as refracture rate, mortality, and initiation and persistence with osteoporosis treatment.

2. Evaluation of quality of life measures among individuals who attend FLS and receive any type of intervention, including pharmacological and non-pharmacological interventions, and compare this to individuals who choose not to attend FLS.

3. Identify optimal service models to improve fracture prevention for individuals who are living in residential and nursing care settings, or those living in their own homes who are receiving maximum levels of support from social services.

4. To explore the feasibility and effectiveness of cascade screening strategies promoted by FLS, whereby the children of elderly fragility fracture patients who are aged 50 years or over themselves are encouraged to seek a bone health assessment from their primary care provider.

\section{Disclosures}

Paul Mitchell (PM): PM has undertaken consultancy for governments, national and international osteoporosis societies, healthcare professional organisations and private sector companies relating to systematic approaches to fragility fracture care and prevention since 2005.

Cyrus Cooper (CC): CC has received consulting fees and honoraria from Amgen, Danone, Eli Lilly, GSK, Medtronic, Merck, Nestle, Novartis, Pfizer, Roche, Servier, Shire, Takeda, and UCB.

Masaki Fujita (MF): MF has no disclosures relating to this work. MF is the Science Project Coordinator of the International Osteoporosis Foundation.

Philippe Halbout (PH): PH has no disclosures relating to this work. PH is the Chief Executive Officer of the International Osteoporosis Foundation.

Kristina Åkesson (KA): KA has no disclosures relating to this work. KA has received consultancy fees from Amgen, Eli Lilly, Renapharma and UCB.

Matthew Costa (MC): $\mathrm{MC}$ has no disclosures relating to this work.

Karsten Dreinhöfer (KD): KD has undertaken consultancy for governments, national osteoporosis societies, healthcare professional organisations and private sector companies relating to systematic approaches to fragility fracture care and prevention since 2004.

David Marsh (DM): DM has no disclosures relating to this work.

Joon-Kiong Lee (JKL): JKL has no disclosures relating to this work.

Ding-Cheng (Derrick) Chan (DCC): DCC has no disclosures relating to this work. DCC is a consultant for Amgen.

M. Kassim Javaid (MKJ): In last five years, MKJ received honoraria, unrestricted research grants, travel and/or subsistence expenses from Amgen, Lilly UK, Internis, Consilient Health, Zebra Medical Vision, Kyowa Kirin Hakin and UCB. 


\section{Acknowledgements}

The Asia Pacific Fragility Fracture Alliance (APFFA) wishes to acknowledge Amgen Asia for their provision of financial support to fund a secretariat function, event management and communication activities leading up to the formation of the Alliance, noting Amgen Asia had no role in the development of this publication. 


\section{References}

Papers of particular interest, published recently, have been highlighted as:

- Of importance

•• Of major importance

1. United Nations Department of Economic and Social Affairs Population Division (2017) World Population Prospects: The 2017 Revision, Key Findings and Advance Tables. Working Paper No. ESA/P/WP.248. New York.

2. United Nations Department of Economic and Social Affairs Population Division (2017) World Population Prospects: Volume II: Demographic Profiles 2017 Revision (ST/ESA/SER.A/400). New York 3. France24. In historic first, G20 weighs ageing as global risk. 2019. https://www.france24.com/en/20190609-historic-first-g20-weighs-ageing-global-risk Accessed 29 Sep 2019.

4. Cooper C, Ferrari S. IOF Compendium of Osteoporosis. In Harvey N, Dennison E, editors. 1st ed. International Osteoporosis Foundation, Nyons. 2019.

5. United Nations Economic and Social Commission for Asia and the Pacific (2016) 2016 ESCAP population data sheet. Social Development Division, Economic and Social Commission for Asia and the Pacific (ESCAP), Bangkok, Thailand.

6. Si L, Winzenberg TM, Jiang $Q$ et al. Projection of osteoporosis-related fractures and costs in China: 2010-2050. Osteoporos Int. 2015 26;1929-1937.

7. Hernlund E, Svedbom A, Ivergard M et al. Osteoporosis in the European Union: medical management, epidemiology and economic burden. A report prepared in collaboration with the International Osteoporosis Foundation (IOF) and the European Federation of Pharmaceutical Industry Associations (EFPIA). Arch Osteoporos. 2013;8:136.

8. United Nations Department of Economic and Social Affairs Population Division (2015) World Population Ageing. United Nations, New York.

9. Zerbini CA, Szejnfeld VL, Abergaria BH et al. Incidence of hip fracture in Brazil and the development of a FRAX model. Arch Osteoporos. 2015;10:224.

10. Burge R, Dawson-Hughes B, Solomon DH et al. Incidence and economic burden of osteoporosisrelated fractures in the United States, 2005-2025. J Bone Miner Res. 2007;22:465-475.

11. Kanis JA, Johnell $\mathrm{O}$, De Laet $\mathrm{C}$ et al. A meta-analysis of previous fracture and subsequent fracture risk. Bone. 2004;35:375-382.

12. Klotzbuecher CM, Ross PD, Landsman PB et al. Patients with prior fractures have an increased risk of future fractures: a summary of the literature and statistical synthesis. J Bone Miner Res. 2000;15:721-739.

13. Gallagher JC, Melton L, Riggs BL, Bergstrath E. Epidemiology of fractures of the proximal femur in Rochester, Minnesota. Clin Orthop Relat Res. 1980;163-171.

14. Port L, Center J, Briffa NK et al. Osteoporotic fracture: missed opportunity for intervention. Osteoporos Int. 2003;14:780-784.

15. McLellan A, Reid D, Forbes K et al. Effectiveness of Strategies for the Secondary Prevention of Osteoporotic Fractures in Scotland (CEPS 99/03). NHS Quality Improvement Scotland. 2004.

16. Edwards BJ, Bunta AD, Simonelli $C$ et al. Prior fractures are common in patients with subsequent hip fractures. Clin Orthop Relat Res. 2007;461:226-230.

17. - Harvey NC, McCloskey EV, Mitchell PJ et al. Mind the (treatment) gap: a global perspective on current and future strategies for prevention of fragility fractures. Osteoporos Int. 2017;28:1507-1529. 
This paper provides a comprehensive summary of the therapeutic evidence base specifically in the context of secondary fracture prevention.

18. Harvey NC, McCloskey EV. Gaps and solutions in bone health: A global framework for improvement. In Misteli L, Laverty C, Stenmark J, editors. World Osteoporosis Day Thematic Report. International Osteoporosis Foundation, Nyon. 2016.

19. Lih A, Nandapalan H, Kim M et al. Targeted intervention reduces refracture rates in patients with incident non-vertebral osteoporotic fractures: a 4-year prospective controlled study. Osteoporos Int. 2011;22:849-858.

20. Bogoch ER, Elliot-Gibson V, Beaton DE et al. Effective initiation of osteoporosis diagnosis and treatment for patients with a fragility fracture in an orthopaedic environment. J Bone Joint Surg Am. 2006;88:25-34.

21. McLellan AR, Gallacher SJ, Fraser M, McQuillian C. The fracture liaison service: success of a program for the evaluation and management of patients with osteoporotic fracture. Osteoporos Int. 2003;14:1028-1034.

22. Dell R, Greene D, Schelkun SR, Williams K. Osteoporosis disease management: the role of the orthopaedic surgeon. J Bone Joint Surg Am. 2008;90:Suppl 4:188-194.

23. Ganda K, Puech M, Chen JS et al. Models of care for the secondary prevention of osteoporotic fractures: a systematic review and meta-analysis. Osteoporos Int. 2013;24:393-406.

24. - Ganda K, Mitchell PJ, Seibel MJ. Models of Secondary Fracture Prevention: Systematic Review and Meta-analysis of Outcomes. In Seibel MJ, Mitchell PJ, editors. Secondary Fracture Prevention: An International Perspective. San Diego: Elsevier; 2019. pp 33-62. This recent meta-analysis provides an update on the impact of Fracture Liaison Services as a function of the intensity of the service model.

25. Seibel MJ, Mitchell PJ, editors. Secondary Fracture Prevention: An International Perspective. San Diego: Elsevier; 2019.

26. Wu CH, Tu ST, Chang YF et al. Fracture liaison services improve outcomes of patients with osteoporosis-related fractures: A systematic literature review and meta-analysis. Bone. 2018;111:92100.

27. $\bullet$ Javaid MK, Kyer C, Mitchell PJ et al. Effective secondary fracture prevention: implementation of a global benchmarking of clinical quality using the IOF Capture the Fracture ${ }^{\circledR}$ Best Practice Framework tool. Osteoporos Int. 2015;26:2573-2578. This paper illustrates the effectiveness of the IOF Capture the Fracture ${ }^{\circledR}$ Programe Best Practice Framework as a tool to benchmark performance of FLS globally.

28. $\quad \bullet$ Javaid MK, Vasilakis N, Dickinson R et al. Fracture Liaison Service Database Annual report December 2018: Achieving effective service delivery by Fracture Liaison Services. Royal College of Physicians, London. 2018. The UK national FLS Database is the first such database in the world. This report could provide colleagues in other countries with insights on how to establish a national FLS Database in their own countries.

29. Majumdar SR, McAlister FA, Johnson JA et al. Comparing Strategies Targeting Osteoporosis to Prevent Fractures After an Upper Extremity Fracture (C-STOP Trial): A Randomized Controlled Trial. J Bone Miner Res. 2018;33:2114-2121

30. McAlister FA, Ye C, Beaupre LA. Adherence to osteoporosis therapy after an upper extremity fracture: a pre-specified substudy of the C-STOP randomized controlled trial. Osteoporos Int. 2019;30:127-134.

31. Black DM, Cummings SR, Karpf DB et al. Randomised trial of effect of alendronate on risk of fracture in women with existing vertebral fractures. Lancet 1996;348:1535-41.

32. van den Berg P, van Haard PMM, Geusens PP et al. Challenges and opportunities to improve fracture liaison service attendance: fracture registration and patient characteristics and motivations. Osteoporos Int. 2019;30:1597-1606. 
33. Osteoporosis New Zealand. Bone Care 2020: A systematic approach to hip fracture care and prevention for New Zealand. Wellington. 2012.

34. Accident Compensation Corporation, Ministry of Health, Health Quality \& Safety Commission New Zealand, New Zealand Government. Live Stronger for Longer: Prevent falls and fractures. 2019. http://livestronger.org.nz/. Accessed 29 Sep 2019.

35. Accident Compensation Corporation, Ministry of Health, Health Quality \& Safety Commission New Zealand. Falls \& fractures outcomes framework: Supporting quality improvement across the health system to reduce harm from falls. 2019.

https://public.tableau.com/profile/hqi2803\#!/vizhome/FallsFracturesOutcomesFramework/Landing. Accessed 29 Sep 2019.

36. - Chang L-Y, Tsai K-S, Peng J-K, et al. The development of Taiwan Fracture Liaison Service network. Osteoporos Sarcopenia. 2018;4:47-52. The approach to drive widespread implementation of the FLS model throughout Taiwan is an example of best prcatice which could be replicated elsewhere. 37. International Osteoporosis Foundation. IOF Capture the Fracture : Map of Best Practice. 2019. https://capturethefracture.org/map-of-best-practice. Accessed 29 Sep 2019.

38. International Osteoporosis Foundation (2017) Capture the Fracture : Taiwanese society reports successes in secondary fracture prevention. 2017. https://www.capturethefracture.org/taiwanesesociety-reports-successes-secondary-fracture-prevention. Accessed 29 Sep 2019.

39. Mitchell PJ. Best practices in secondary fracture prevention: fracture liaison services. Curr Osteoporos Rep. 2013;11:52-60.

40. Gittoes N, McLellan AR, Cooper A et al. Effective Secondary Prevention of Fragility Fractures: Clinical Standards for Fracture Liaison Services. National Osteoporosis Society, Camerton. 2015.

41. Royal Osteoporosis Society. FLS Implementation Toolkit. 2019. https://theros.org.uk/healthcareprofessionals/fracture-liaison-services/implementation-toolkit/. Accessed 29 Sep 2019.

42. Royal College of Physicians. Fracture Liaison Service Database (FLS-DB). 2019.

https://www.rcplondon.ac.uk/projects/fracture-liaison-service-database-fls-db. Accessed 29 Sep 2019

43. Royal College of Physicians. Fracture Liaison Service Database (FLS-DB) facilities audit - FLS breakpoint: opportunities for improving patient care following a fragility fracture. Royal College of Physicians, London. 2016.

44. Royal College of Physicians. FLS-DB clinical audit: Identifying high-quality care in the NHS for secondary fracture prevention. Royal College of Physicians, London. 2017.

45. Axelsson $\mathrm{KF}$, Jacobsson $\mathrm{R}$, Lund $\mathrm{D}$, Lorentzon $\mathrm{M}$. Effectiveness of a minimal resource fracture liaison service. Osteoporos Int. 2016;27:3165-3175.

46. Andreasen C, Solberg LB, Basso T et al. Effect of a Fracture Liaison Service on the Rate of Subsequent Fracture Among Patients With a Fragility Fracture in the Norwegian Capture the Fracture Initiative (NoFRACT): A Trial Protocol. JAMA Netw Open. 2018;1:e185701.

47. Borgen $T$, Bjornerem $A$, Solberg $L B$ et al. High prevalence of vertebral fractures and low trabecular bone score in patients with fragility fractures: A cross-sectional sub-study of NoFRACT. Bone. 2019;122:14-21.

48. Tei RMH, Ramlau-Hansen CH, Plana-Ripoll O et al. OFELIA: Prevalence of Osteoporosis in Fragility Fracture Patients. Calcif Tissue Int. 2019;104:102-114.

49. Tei RMH, Plana-Ripoll O, Brink O, Langdahl BL. An Optimised Fracture Liaison Service Model: Maintained Diagnostic Sensitivity Despite Reduced Number of Diagnostic Tests Performed. Calcif Tissue Int. 2019;104:641-649.

50. Akesson K, Marsh D, Mitchell PJ et al. Capture the Fracture: a Best Practice Framework and global campaign to break the fragility fracture cycle. Osteoporos Int. 2013;24:2135-2152.

51. International Osteoporosis Foundation. Capture the Fracture ${ }^{\circledR}$ Programme website. 2019. https://www.capturethefracture.org/. Accessed 29 Sep 2019. 
52. International Osteoporosis Foundation. Capture the Fracture Website - Mentorship page. 2019. https://capturethefracture.org/mentorship-programme. Accessed 29 Sep 2019.

53. International Osteoporosis Foundation. IOF Global Patient Charter. 2017.

https://www.iofbonehealth.org/iof-global-patient-charter. Accessed 29 Sep 2019.

54. Center for Medical Technology Policy. Strategic Roadmap to Prevent Secondary Fractures.

Baltimore. 2017.

55. American Society for Bone and Mineral Research. Patients 65 Years of Age or Older Who Experience a Hip or Spine Fracture Should be Treated for Osteoporosis, Says Global Coalition of Bone Health Experts and Patient Advocacy Organizations. 2018.

http://www.asbmr.org/about/pressreleases/detail.aspx?cid=8281a027-f61f-446f-ba1f-77b4a2222d8e. Accessed 29 Sep 2019.

56. $\quad-\bullet$ Dreinhofer KE, Mitchell PJ, Begue T et al. A global call to action to improve the care of people with fragility fractures. Injury. 2018;49:1393-1397. This Global Call to Action received an unprecedented level of endorsement from healthcare professional organisations throughout the world, and may serve as a catalyst to stimulate formtion of national multi-sector, multidisciplinary alliances to expedite national policy change.

57. International Osteoporosis Foundation (2018) Launch of multidisciplinary alliance aiming to curb Asia Pacific's fragility fractures. https://www.iofbonehealth.org/news/launch-multidisciplinary-allianceaiming-curb-asia-pacific\%E2\%80\%99s-fragility-fractures. Accessed 19 Sep 2019.

\section{Tables and legends for figures}

Figure 1. Old-age dependency ratios for the world and major world regions for the period 1950 to 2100 [2]

From World Population Prospects: Volume II: Demographic Profiles 2017 Revision. ST/ESA/SER.A/400, by Department of Economic and Social Affairs, Population Division, ${ }^{\circ} 2017$ United Nations. Reprinted with the permission of the United Nations.

Figure 2. The IOF Capture the Fracture ${ }^{\circledR}$ Map of Best Practice to June 2019 [36]

Reproduced with kind permission of the International Osteoporosis Foundation 